\title{
Left ventricular dysfunction and reversible pulmonary hypertension secondary to severe pneumonia in a background of sepsis: a case report and review of the literature
}

\author{
Elaine N. Gitonga ${ }^{1}$, Junwu Wang ${ }^{1}$, Shengwei $\mathrm{Yu}^{1}, \mathrm{Na} \mathrm{Wu}^{2}$, Haitao Shen ${ }^{1}$ \\ ${ }^{1}$ Department of Emergency Medicine, Shengjing Hospital of China Medical University, Shenyang, China; ${ }^{2}$ Department of Endocrinology, Shengiing \\ Hospital of China Medical University, Shenyang, China \\ Correspondence to: Dr. Haitao Shen. Department of Emergency Medicine, Shengjing Hospital of China Medical University, 36 Sanhao Street, \\ Shenyang, China. Email: shenht@sj-hospital.org.
}

\begin{abstract}
Pneumonia is a well-recognized respiratory infection associated with substantial morbidity and mortality. Despite its effects on the respiratory system, pneumonia can cause or exacerbate cardiovascular complications through various mechanisms. The two main mechanisms that are described in this case report are hypoxia-induced pulmonary hypertension and the effect of sepsis on the cardiovascular system. Pulmonary hypertension $(\mathrm{PH})$ is a disease characterized by raised pulmonary arterial pressure due to a progressive increase in pulmonary vascular resistance, inevitably leading to right ventricular (RV) afterload. For our case, the situation was complicated by sepsis, which further worsened the myocardial function causing left ventricular hypertrophy and left ventricular dysfunction. The main goal of this case report is to highlight the fact that cardiovascular events due to pneumonia are a potential complication even in young patients who are without any comorbidities. We present a case of a 14-year-old patient who presented with symptoms of cough, hemoptysis, fever, chest pain, and dyspnea. After the necessary investigations, he was diagnosed with severe pneumonia, sepsis, moderate $\mathrm{PH}$, and left ventricular dysfunction. The treatment course was focused on stabilizing the patient by oxygen supplementation, treating the underlying cause with the use of antibiotics, and decreasing the already raised arterial pressures through vasodilator therapy. After the patient went through the proper course of treatment, there was a marked improvement in his general condition.Cardiac complications due to pneumonia are potential complications even in relatively young patients who have no noted comorbidities. Clinicians should be aware of these potentially fatal complications of pneumonia and appreciate the significance of this association for timely recognition, diagnosis, and management of these complications.
\end{abstract}

Keywords: Pulmonary hypertension (PH); severe pneumonia; sepsis; sepsis-induced cardiomyopathy; left ventricular dysfunction

Submitted Apr 21, 2020. Accepted for publication Sep 08, 2020.

doi: 10.21037/apm-20-1198

View this article at: http://dx.doi.org/10.21037/apm-20-1198

\section{Introduction}

Pneumonia is a common condition associated with substantial morbidity and mortality, irrespective of age, and is one of the major common occurrences in the Emergency Department and the ICU. Pneumonia not only affects the respiratory system but it has come to be discovered that it also directly or indirectly affects the cardiovascular system by causing cardiac events like acute myocardial infarction, heart failure, arrhythmias, stroke, or worsening of preexisting cardiac conditions. Several mechanisms have been reported to be involved with one being increased pulmonary vascular resistance due to hypoxia and as a result, an increase in pulmonary arterial pressure causing 
pulmonary hypertension $(\mathrm{PH}) . \mathrm{PH}$ is a disease characterized by an increase in pulmonary arterial pressure and resultant vascular remodeling (thickening of the vessel walls and narrowing of the vessel lumens). Usually, the pulmonary vasculature in itself is a low-pressure system with the pressure of the pulmonary artery (mPAP) falling at around $25 \mathrm{mmHg}$ at rest and $30 \mathrm{mmHg}$ during activity. When pulmonary pressures go beyond the mentioned ranges, then it is considered PH. Through recent years, much research has gone into understanding the etiology and effects of PH. PH can come about due to primary causes (idiopathic $\mathrm{PH})$ or secondary due to conditions that bring about a rise in pulmonary arterial pressure. The World Health Organization (WHO) managed to classify the causes of $\mathrm{PH}$ based on the etiology and pathophysiology of $\mathrm{PH}$ :

* Group 1: primary or idiopathic PH (heritable, and HIV-associated; systemic sclerosis and other connective tissue diseases).

* Group 2: PH due to left-sided heart disease (including systolic and diastolic dysfunction and valvular heart disease).

* Group 3: PH associated with lung respiratory disease/ or hypoxia.

* Group 4: PH due to chronic thrombotic or embolic disease.

* Group 5: due to multiple multifactorial etiologies [including metabolic, systemic, and hematologic disorders (sickle cell disease), and others].

In our case, $\mathrm{PH}$ was of an acute setting. However, if we were to use the $\mathrm{WHO}$ grouping of $\mathrm{PH}$, our patient would fall under WHO group 3, which is characterized by hypoxemia secondary to lung respiratory disease (which in our case was severe pneumonia). Pneumonia is characterized by the accumulation of exudate in the alveolar spaces, resulting in acute hypoxemia. Persistent hypoxemia due to alveolar consolidation affects the normal ventilation/ perfusion (V/Q) homeostasis, which in turn leads to an increase in pulmonary resistance, raised pulmonary arterial pressure, increasing right ventricular (RV) afterload. Persistent inflammation such as in the case of sepsis has also been linked to being a cause of generalized myocardial dysfunction.

The main goal of this case report is to highlight the fact that cardiovascular events due to pneumonia are a potential complication and may arise in a relatively young patient who has no other comorbidities. Therefore clinicians must not be short-sighted when dealing with a patient with severe pneumonia with worsening symptoms despite treatment.
Patients with severe pneumonia should be considered high-risk patients and should be diligently monitored for any possibility of cardiac events that may arise due to the infection. We present the following article in accordance with the CARE reporting checklist (available at http:// dx.doi.org/10.21037/apm-20-1198).

\section{Methods}

We present the case of a 14-year-old patient who was diagnosed with severe pneumonia, sepsis, moderate $\mathrm{PH}$, and left ventricular dysfunction to highlight the fact that cardiovascular events due to pneumonia are a potential complication even in young patients who are without any comorbidities. Then we collect the literature about left ventricular dysfunction and pulmonary hypertension to discuss about the mechanisms.

All procedures performed in studies involving human participants were in accordance with the ethical standards of the institutional and/or national research committee(s) and with the Helsinki Declaration (as revised in 2013). Written informed consent was obtained from the patient's father for publication of this case report and any accompanying images. A copy of the written consent is available for review by the Editor-in-Chief of the journal upon request.

\section{Results}

\section{Case presentation and ethical statement}

A 14-year-old male was referred to us from a peripheral hospital, where he had then presented with complaints of cough, bloody sputum, and nausea. At the time, he was afebrile and had no difficulty in breathing. A chest CTScan returned typical results. Nonetheless, he was started on a Cefaclor. Four days during treatment, his general condition worsened; it was noted that he was febrile with the highest temperature at around $39.0 \mathrm{deg}$. Celsius, with associated chills. They then added Clindamycin, Brufen, and Ganciclovir to his treatment regimen. A day later, the patient started having trouble breathing, which was worse when lying flat (Orthopnea), and he was also noted to be confused. However, despite treatment with now two antibiotics and antiviral coverage, his general condition continued to deteriorate. A repeat chest CT scan was then done, and it showed multilobar pneumonia that was more severe on the left upper lobe. An echocardiogram was also done and showed moderate PH. Due to his 


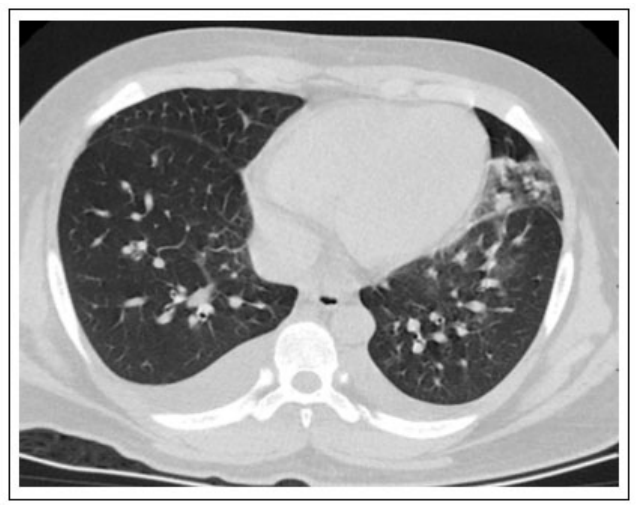

Figure 1 Chest CT scans on admission (day 1 of admission): bilateral pulmonary infiltrates, bilateral pleural effusion, enlarged mediastinal lymph nodes, and calcification on the right lobe of the liver. There were no noted pulmonary emboli or any pulmonary artery obstruction.

worsening clinical state despite all the medical intervention, he was referred to our EICU for admission and further management.

He presented at our EICU with complaints of cough, difficulty in breathing that was worse when lying flat, bloody sputum, fevers and chills, chest pains which were pleuritic in nature, chest tightness, nausea, and decreased urine output. It should be noted that during his presentation, we did not observe any bouts of confusion that were initially stated.

Before this admission, his past medical history was uneventful; he was a healthy, fit young man, with no history of diabetes, hypertension, asthma, or any other disease. His familial history was also unremarkable.

Physical examination revealed that our patient was tachypneic at 25 breaths/min and $\mathrm{SPO}_{2}$ of $76 \%$ on room air, tachycardic at $130 \mathrm{bpm}$, hypertensive at 197/120 $\mathrm{mmHg}$. There was no noted cyanosis, no scleral jaundice, or pedal edema noted. On auscultation of the chest, there were normal bilateral vesicular breath sounds. There were bilateral crepitations which were more pronounced on the left upper lobe. On his cardiovascular exam, s1s2 were heard with no noted murmur. The jugular venous pulsation was regular. There was no noted parasternal heave. His abdominal examination was routine.

Their full blood count showed slightly elevated white blood cells at $9.74 \times 10^{9} / \mathrm{L}$ and an elevated CRP of 36.80 , giving us an inflammatory picture. BGA: $\mathrm{PH} 7.41, \mathrm{PaCO} 2$ $31.4 \mathrm{mmHg}, \mathrm{PaO} 2,85 \mathrm{mmHg}$. Neurohumoral marker: NT-pro BNP was elevated at $3630 \mathrm{ng} / \mathrm{L}$; nevertheless, the cardiac enzymes were normal. Liver function tests were normal. In the Coagulation profile, $\mathrm{d}$-dimers were elevated at $632 \mu \mathrm{g} / \mathrm{L}(1,2)$. Ferritin levels were normal. Urinalysis showed increased RBC and protein. HIV test and TB-negative. Autoimmune, autoantibody, and immunologic studies were all normal. The flow cytometry results of lymphocytes were normal. Positive mycoplasma pneumoniae IgG. Blood cultures were done; however, there was no noted growth. Due to our patient's persistent high blood pressure, tests to rule out secondary causes of hypertension were ordered (UEC's, 24-hour urine collection protein test and VMA, serum TSH, ACTH and Cortisol, plasma aldosterone to renin activity, renal artery Doppler ultrasound) which were all normal.

\section{CT-scan of the chest}

Bilateral pulmonary infiltrates, more severe on the left upper lobe, bilateral pleural effusion, enlarged mediastinal lymph nodes, and calcification on the right lobe of the liver. There were no noted pulmonary emboli or any pulmonary artery obstruction (Figure 1). Twelve-lead ECG- sinus tachycardia, no changes in ST-segment or T waves (Figure 2).

\section{Transthoracic echocardiogram}

Moderate $\mathrm{PH}$, moderate tricuspid regurgitation, and normal contraction of the ventricles, ejection fraction was normal at $63 \%$ (Figure 3).

The necessary investigations were done a diagnosis of moderate $\mathrm{PH}$ and left ventricular hypertrophy in a patient with severe pneumonia, and sepsis was made.

All procedures performed in studies involving human participants were in accordance with the ethical standards of the institutional and/or national research committee(s) and with the Helsinki Declaration (as revised in 2013). Written informed consent was obtained from the patient.

\section{Treatment}

The patient's treatment course was focused on stabilizing the patient through the management of presenting symptoms, treating the underlying cause of the PAH which was pneumonia and sepsis, and also decreasing the already increasing pulmonary arterial pressure through the use of vasodilator therapy (Table 1). Our patient presented in a hypoxic state with oxygen saturation of $76 \%$ breathing room air. Respiratory stabilization through supplemental oxygen was crucial. Ventilatory support was initiated on $\mathrm{O}_{2} 5 \mathrm{~L} / \mathrm{min}$ via a non-rebreather mask. The patient's 


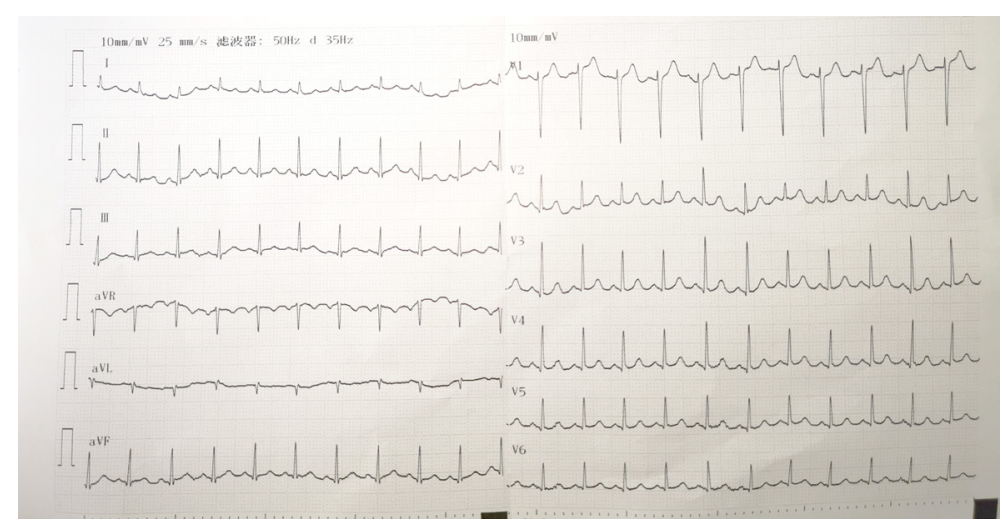

Figure 2 ECG (day 1 of admission)-sinus tachycardia, no changes in ST-segment or T waves.
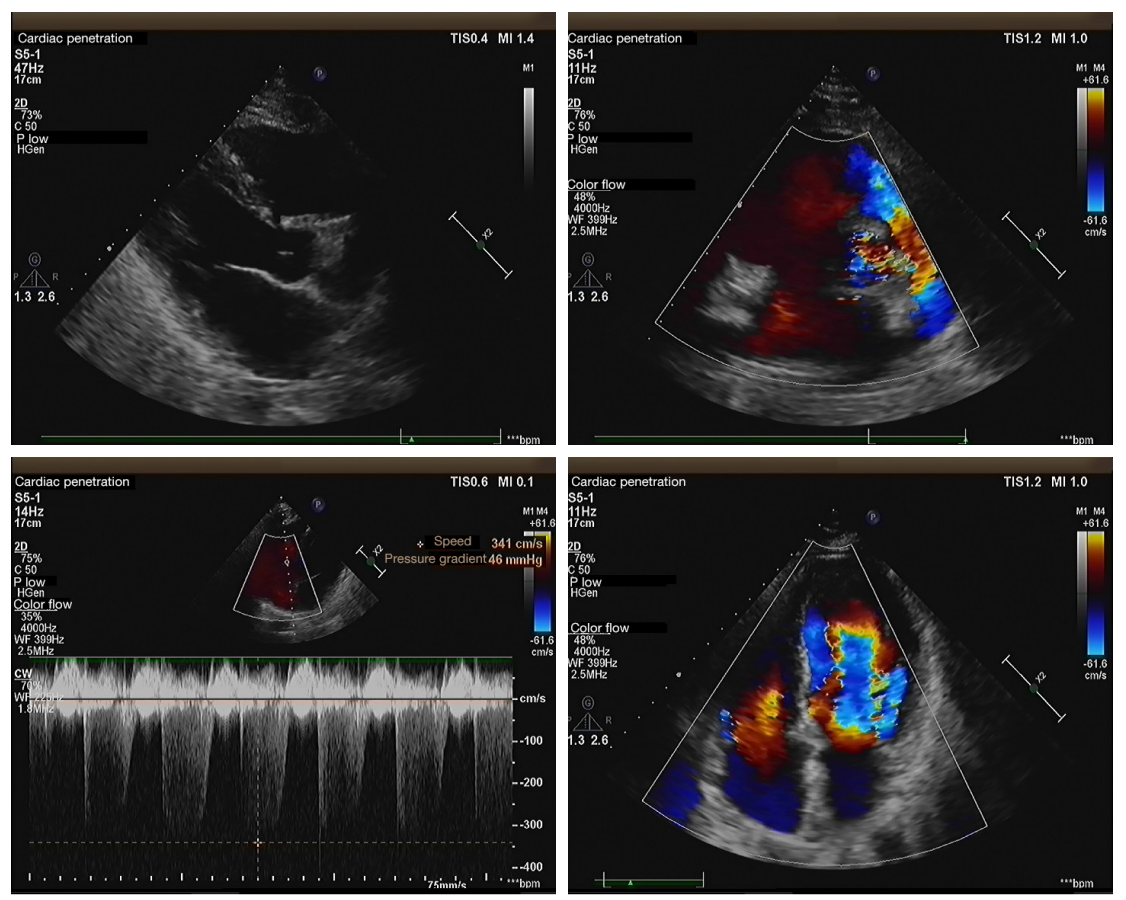

Figure 3 TTE (day 1): moderate pulmonary hypertension (internal diameter of the pulmonary artery-23, estimation of pulmonary systolic pressure-52 $\mathrm{mmHg}$ ), moderate tricuspid regurgitation (peak speed-3.7 m/s) and normal contraction of both ventricles, ejection fraction was normal at $63 \%$.

oxygen saturation recovered to $92 \%$. Treatment for severe pneumonia was also started on Ertapenem $1 \mathrm{~g}$ IV OD. During antibiotic treatment, a mycoplasma pneumoniae antibody IgG was performed and was positive. The patient was then started on a macrolide (azithromycin initial dose $500 \mathrm{mg}$ then a continuation dose of $250 \mathrm{mg}$ od) for antimycoplasma coverage. Since it was during winter and the peak period for influenza, he was put on antiviral prophylaxis. He was started on oseltamivir phosphate, an antiviral neuraminidase inhibitor used for the treatment and prophylaxis of infection with influenza viruses $\mathrm{A}$ and $\mathrm{B}$ and Arbidol hydrochloride, a broad-spectrum antiviral that has demonstrated activity against several enveloped and nonenveloped viruses with immunomodulatory properties used in the prevention of severe pneumonia and virus-associated cytokine dysregulation induced by influenza viruses. 
Table 1 Summary of our patient's clinical course

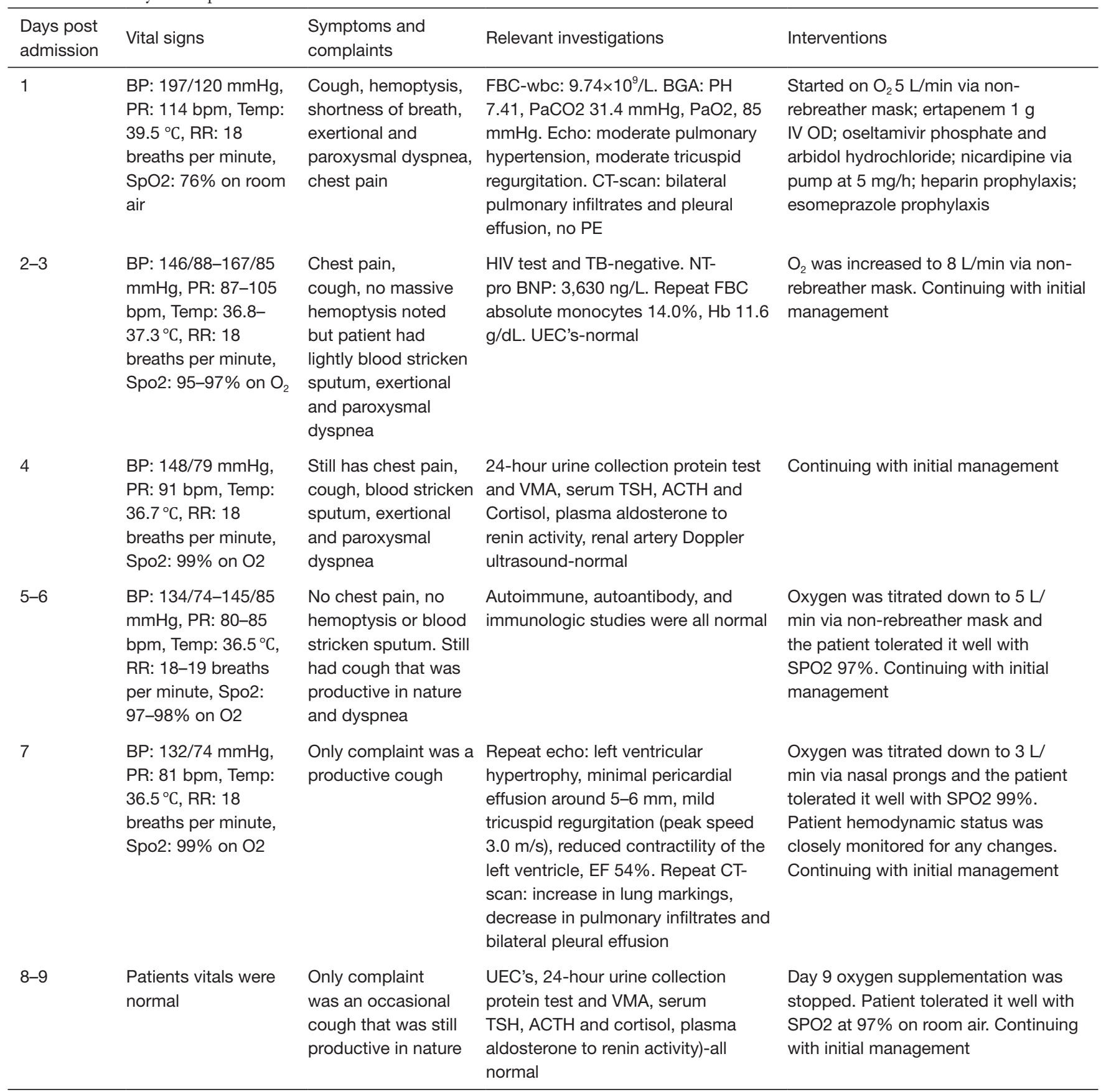

Table 1 (continued) 
Table 1 (continued)

\begin{tabular}{|c|c|c|c|c|}
\hline $\begin{array}{l}\text { Days post } \\
\text { admission }\end{array}$ & Vital signs & $\begin{array}{l}\text { Symptoms and } \\
\text { complaints }\end{array}$ & Relevant investigations & Interventions \\
\hline $10-12$ & $\begin{array}{l}\text { BP: } 140 / 72 \mathrm{mmHg} \text {, } \\
\text { PR: } 78 \text { bpm, Temp: } \\
36.9^{\circ} \mathrm{C}, \mathrm{RR}: 18 \\
\text { breaths per minute, } \\
\text { Spo2: } 99 \% \text { on room } \\
\text { air }\end{array}$ & $\begin{array}{l}\text { Only complaint } \\
\text { was an occasional } \\
\text { productive cough }\end{array}$ & $\begin{array}{l}\text { MP IgG mycoplasma pneumoniae } \\
\text { antibody IgG positive. True PCT } \\
\text { procalcitonin } 0.059 \text { showing } \\
\text { moderate inflammatory reaction. } \\
\text { ESR: elevated at } 56\end{array}$ & $\begin{array}{l}\text { Ertapenem, oseltamivir, arbidol, } \\
\text { heparin and esomeprazole were } \\
\text { stopped. Patient was started on oral } \\
\text { azithromycin with initial dose of } 500 \\
\text { mg od due to the positive MP IgG } \\
\text { test. Nicardipine was titrated down } \\
\text { to } 2 \mathrm{mg} / \mathrm{hr} \text {. via infusion pump. Patient } \\
\text { hemodynamic status was closely } \\
\text { monitored for any changes. Continued } \\
\text { on Azithromycin dose of } 250 \mathrm{mg} \text { od }\end{array}$ \\
\hline 13 & $\begin{array}{l}\text { BP: } 134 / 80 \mathrm{mmHg} \text {, } \\
\text { PR: } 75 \text { bpm, Temp: } \\
36.5^{\circ} \mathrm{C}, \mathrm{RR}: 18 \\
\text { breaths per minute, } \\
\text { Spo2: } 100 \% \text { on room } \\
\text { air }\end{array}$ & $\begin{array}{l}\text { Patient only } \\
\text { complaint was a mild } \\
\text { cough. He was stable } \\
\text { and active }\end{array}$ & & $\begin{array}{l}\text { IV nicardipine was stopped and patient } \\
\text { was transitioned to oral nicardipine. } \\
\text { Azithromycin dose of } 250 \mathrm{mg} \text { od }\end{array}$ \\
\hline 14 & $\begin{array}{l}\text { BP: } 136 / 84 \mathrm{mmHg} \text {, } \\
\text { PR: } 75 \text { bpm, Temp: } \\
36.6^{\circ} \mathrm{C}, \mathrm{RR}: 18 \\
\text { breaths per minute, } \\
\text { Spo2: } 99 \% \text { on room } \\
\text { air }\end{array}$ & No complaints & $\begin{array}{l}\text { Echo: normal cardiac function, no } \\
\text { noted abnormality. EF } 64 \%\end{array}$ & $\begin{array}{l}\text { Oral nicardipine was stopped. Close } \\
\text { observation of patient hemodynamic } \\
\text { status }\end{array}$ \\
\hline 15 & $\begin{array}{l}\text { Patients vitals were } \\
\text { normal }\end{array}$ & No complaints & & Patient was discharged \\
\hline
\end{tabular}

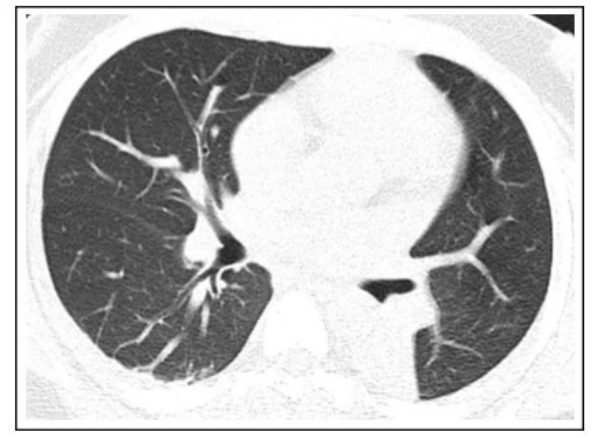

Figure 4 Chest CT scans a week after admission (day 7): decreased expansion of the left lower lobe, increased lung markings in bilateral lower lobes of the lungs, overall bilateral decrease in the pulmonary infiltrates, decrease in bilateral pleural effusion, minimal pericardial effusion, reduced mediastinal lymphadenopathy as compared to the initial chest CT.
Coagulopathy is one of the complications in patients with severe pneumonia and since the patient was experiencing exertional dyspnea he was unable to ambulate, therefore it was necessary to start him on heparin prophylaxis $(5,000$ units SC BD) for the prevention of thrombus formation. $\mathrm{He}$ was also started on esomeprazole $40 \mathrm{mg}$ IV as prophylaxis for upper G.I. bleed. Vasodilator therapy through the use of calcium channel blockers was essential for the reduction of vasoconstriction and as such the reduction of the high arterial pressures. An infusion of nicardipine via a pump at $5 \mathrm{mg} / \mathrm{h}$ was initiated, and his blood pressure was continuously monitored. Serial high-resolution CTscans and transthoracic echocardiograms were performed to monitor the patient's response to treatment. Chest CTscan a week after admission showed a bilateral decrease in the pulmonary infiltrates (Figure 4), TTE then showed left 

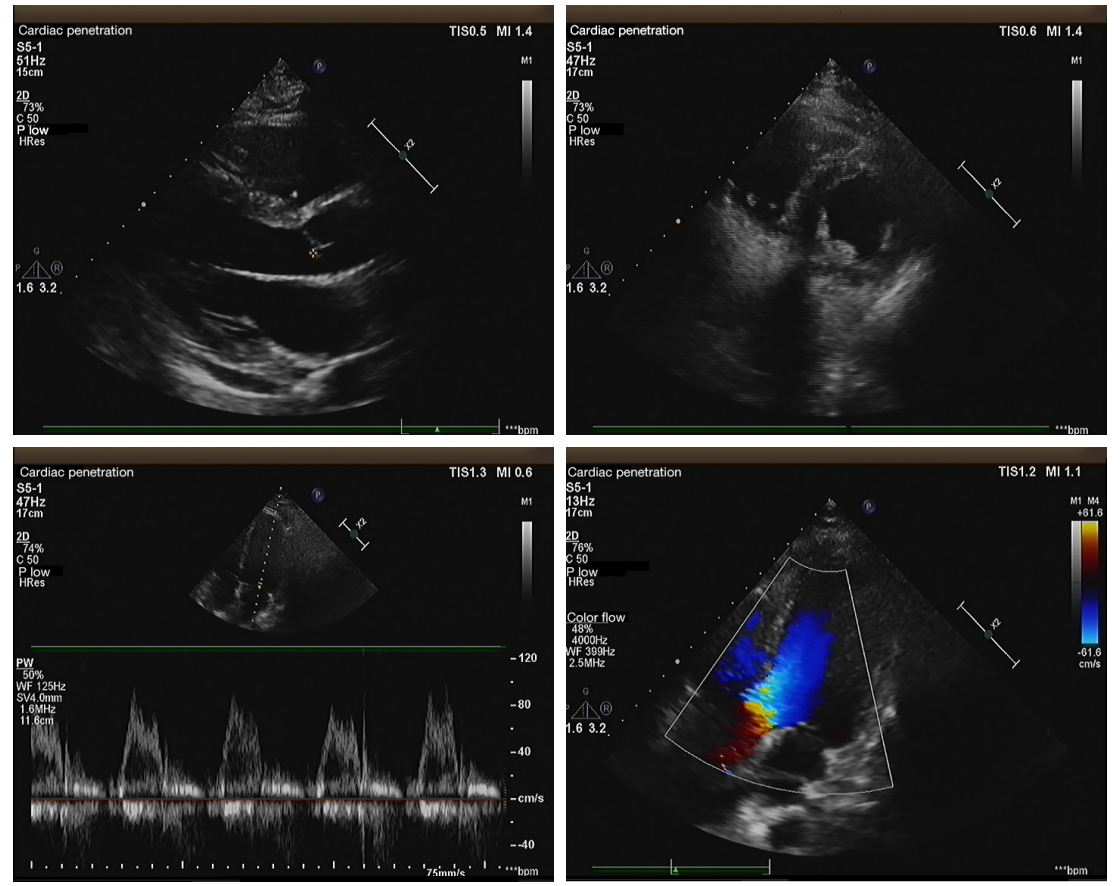

Figure 5 TTE (day 7 after admission): left ventricular hypertrophy, minimal pericardial effusion around 5-6 mm, mild tricuspid regurgitation (peak speed $-3.0 \mathrm{~m} / \mathrm{s}$ ), reduced contractility of the left ventricle, EF-54\%.
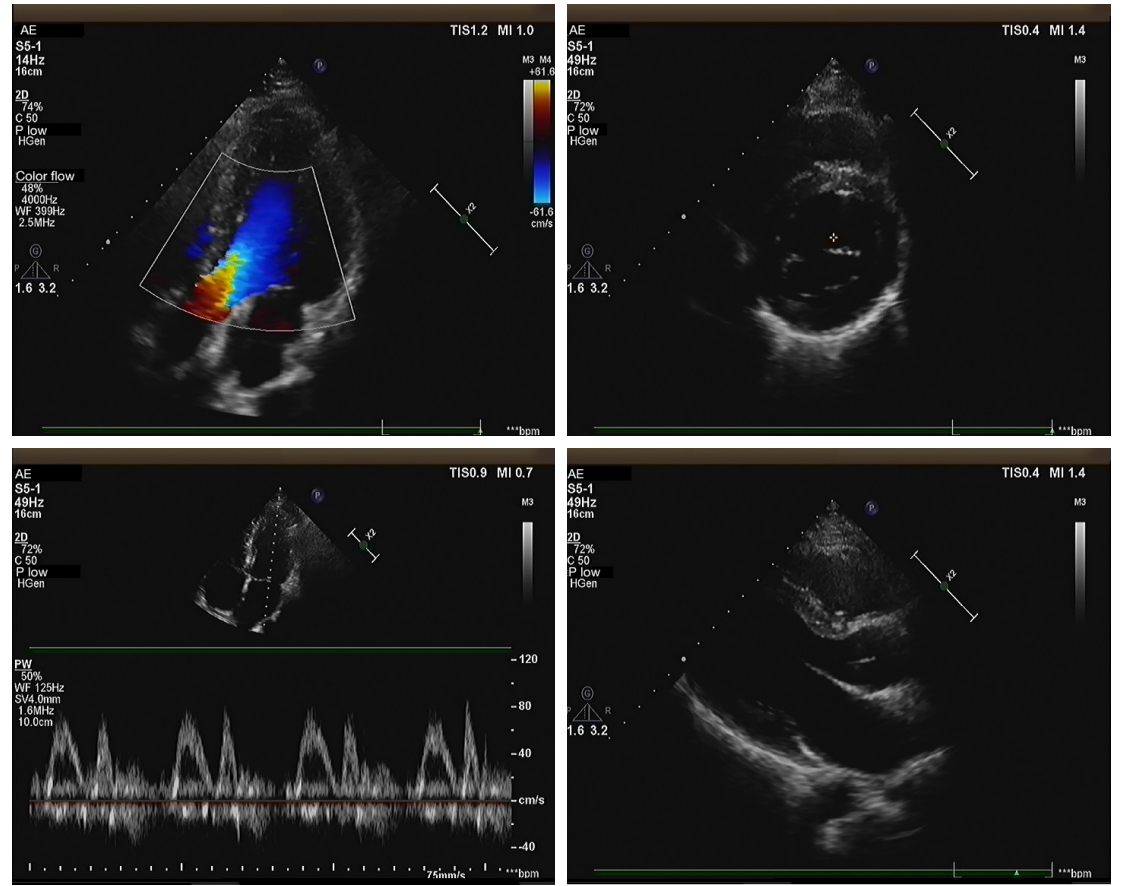

Figure 6 TTE (day 13 after admission): normal cardiac function, no noted abnormality. EF-64\%. 
ventricular hypertrophy and reduced contractility of the left ventricle (Figure 5). As the blood pressure normalized, nicardipine was gradually tapered off and stopped four days before discharge. The TTE then showed a normal cardiac function (Figure 6). The patient remained normotensive for the rest of his admission and during his follow up.

\section{Discussion}

\section{Pneumonia and cardiovascular complications}

Pneumonia is a disease affecting the lung parenchyma causing inflammation of the alveoli leading to inflammatory exudate filling the alveolar spaces. The main causative agents are bacteria, fungi, and viral agents; despite the respiratory tract being always in contact with air that has hundreds to thousands of microorganisms per cubic meter, it is well adapted to defend our bodies from any virulent organism $(3,4)$. Nonetheless, in a case where the host defenses fail, and a virulent factor penetrates through our protective mechanisms, and get to the lung, the inflammatory reaction triggered by the macrophages within the lungs cause histopathological changes within the lung itself and in turn affects the functionality of the lungs leading to hypoxia, and if left untreated may lead to respiratory failure (5-10). Despite respiratory failure due to hypoxemia being one of the most commonly noted complications of pneumonia, pneumonia has also been found to cause other cardiovascular complications or worsening of preexisting cardiac conditions (11).

The main mechanisms responsible for cardiovascular complications have been linked to the patients' demographics (age, presence of comorbidities), pneumonia severity, and systemic response to infection. Hypoxia or respiratory failure causes an increase in pulmonary arterial pressure and also right ventricular afterload (1217). Persistent inflammation as in the case of sepsis causes depression in myocardial function and increases myocardial oxygen demand. An increase of sympathetic nervous system (SNS) activity which is a basic response to inflammation causes increased heart rate and vascular resistances; this causes a decrease in cardiac output and coronary perfusion of the heart. Cardiac rhythm imbalances mainly atrial fibrillation comes about due to the direct effect of disease or inflammation on the cardiac electrical system (18). Inflammatory activity causes prothrombotic changes in the blood and endothelium, resulting in plaque instability and facilitating coronary thrombosis (19). Coronary thrombus formation and plaque instability may lead to plaque rupture which in turn may cause occlusion of coronary or cerebral blood flow causing myocardial infarction or ischemic stroke. Direct myocardial insults by pathogens (both viral and bacterial) may lead to myocardial inflammation, causing myocarditis $(11,20,21)$.

\section{Pathophysiology of hypoxia and PH}

Respiratory failure secondary to hypoxia is usually a result of two main mechanisms. The first one being that during inflammation, the alveoli are filled with inflammatory exudate. The inflammatory exudate reduces the available surface area of the alveoli that are used in gas exchange (the functional residual capacity-FRC). The second mechanism is through V/Q mismatch ( $\mathrm{V}$-ventilation, Q-perfusion). The V/Q ratio is the amount of air reaching the alveoli per minute to the amount of blood reaching the alveoli from the capillaries per minute. Perfusion within the lung is affected by subatmospheric intrapleural pressure and gravity; as a result, perfusion is higher at the lower lobes than at the peak of the lungs. These differences have a significant impact on the V/Q ratio-the V/Q ratio is higher on the apex of the lung than on the base of the lung (22). If the lung is taken as a whole, the mean V/Q ratio is around 0.8. During the acute phase of pneumonia, the pulmonary vascular system continues to maintain blood flow to the affected regions of the lung, and this causes an intrapulmonary shunt (persistent perfusion to the alveoli with inadequate ventilation). In such cases, if there is a low V/Q ratio, it causes a decrease in the $\mathrm{PAO} 2$ which later causes a decrease in the $\mathrm{PaO} 2$ causing hypoxemia.

After chronic exposure to hypoxemia, the lungs have a compensatory mechanism called hypoxic pulmonary vasoconstriction (HPV) or the Euler-Liljestrand mechanism (23). HPV causes the small pulmonary arteries to constrict and reduce blood flow to the areas in the lungs with decreased ventilation and redirect the blood to better-oxygenated areas, and by doing so, optimizing V/Q matching. Persistent HPV leads to vascular remodeling (medial hypertrophy and narrowing of arterial lumens), which in turn causes an increase in pulmonary vascular resistance and concurrently increases pulmonary arterial pressure causing $\mathrm{PH}(12,13)$. The right ventricle as compared to the left ventricle is thin-walled; it can accommodate changes in the preload; however, it is limited in tackling changes in the afterload. As the pulmonary pressures increase, it leads to a decrease in the RV stroke volume and output. The pressure 
causes an increase in the volume within the right ventricle, and the right ventricle has to work harder to pump the extra volume (14-17). The ventricle dilates in response to this. In time, ventricular dilation fails, and this leads to ventricular dysfunction. This mechanism is explained by the FrankStarling rule (24-28). The increased volume and pressures also cause or increase the severity of tricuspid regurgitation.

For our case, the second echo showed signs of left ventricular hypertrophy and worsening of the left ventricular function. These signs can be attributed to the rising of the RV pressure and sepsis. The right and left ventricles are separated by a septum (the interventricular septum). Usually, the septum is bowed towards the right ventricle; this is mainly because the left ventricular enddiastolic pressure is higher than that of the right. Persistent $\mathrm{PH}$ causes an increase in the afterload in the right ventricle. The increased pressure is transmitted to the septum, initially causing flattening of the septum and later causes bowing of the septum towards the left ventricle. The pressure further impairs the function of the left ventricle by reducing the left ventricular diastolic filling and cardiac output. Due to this deficit, the left ventricle compensates, leading to the thickening of the left ventricle.

\section{Diagnosis of PH}

A positive diagnosis of $\mathrm{PH}$ should be undertaken in a stepwise approach. Clinicians need to carefully consider the patient's past medical history and family history. The presenting complaints of $\mathrm{PH}$ may be non-specific ranging from cough, easy fatigability, weakness, syncope, hemoptysis to severe symptoms of right heart failure like pitting edema, and abdominal distention. Physical examination of the patient also helps in showing the underlying cause. Although blood tests are not normally used to diagnose PH they are necessary in that they help give insight on the nature of the cause of the $\mathrm{PH}$ for example blood gas analysis, coagulation profile, screening for connective tissue diseases, HIV, hepatitis, and immunological studies. An electrocardiogram (ECG) is one of the cardinal investigations that should be performed, it may show signs of changes in the heart or abnormal rhythms in the heart's electrical activity caused by pulmonary hypertension e.g., right ventricle hypertrophy by showing right-axis deviation. It should be noted that one may still have pulmonary hypertension even if the ECG is normal. A High-resolution CT-scan of the chest is necessary to give a clear picture of the lungs, and also to rule out any thromboembolic causes of $\mathrm{PH}$. Transthoracic echocardiogram (TTE) is the most important non-invasive diagnostic investigation for $\mathrm{PH}$ and it shows the effects of $\mathrm{PH}$ on the heart, any valvular abnormalities, and the estimated right ventricular (RV) systolic pressure or PAP from continuous wave Doppler. Pulmonary function test (PFT) measures lung volume, capacity, rates of flow, and gas exchange and will show a decreased diffusing capacity (DLCO) in all patients with pulmonary hypertension. A DLCO of less than $45 \%$ is also indicative of a bad general outcome for the patient. Ventilation-perfusion scanning of the lungs may also be done to determine if the cause is suspected to be thromboembolic in nature. Despite TTE being the most commonly used testing modality, it is not the most accurate in assessing the level of hemodynamic impairment in patients. Right-sided heart catheterization (RHC) is the most definitive test for diagnosis and is also useful in assessing responsiveness to treatment. RHC is an invasive test and should only be performed after diagnosis through TTE has been done. Vasoreactivity testing should be performed concurrently with RHC to identify the small minority of patients who may benefit from vasodilator therapy through the use of calcium channel blockers (CCB) (29-32). In 2015 ESC/ERS released guidelines for the diagnosis and the treatment of PAH (Figure 7) $(29,33)$.

\section{Treatment of $\mathrm{PH}$}

The treatment options for pulmonary hypertension depend on the underlying cause of the PAH. Once the cause has been established treatment of the cause should be initiated, for example in our case antibiotics (ertapenem and azithromycin) were initiated for the treatment of pneumonia. General treatment of $\mathrm{PH}$ is mainly symptomatic, with the main focus being in the reduction of the already high pulmonary arterial pressures and also in the reduction of the workload on the right ventricle. Oxygen supplementation in patients with hypoxia should be initiated and oxygen saturation $(\mathrm{SpO} 2)$ should be maintained above $90 \%$. Measures should also be undertaken in patients with nocturnal hypoxemia (obstructive sleep apnea) like lifestyle changes and nocturnal nasal continuous positive airway pressure. Patient hydration status should also be considered, fluid input and urine output should be closely monitored. Caution should be placed when rehydrating patients with subpar right ventricular function. Any anemia and related deficiencies (iron deficiency or vitamin $\mathrm{C}$ deficiency) should be corrected. Loop diuretics are useful to patients with signs of fluid overload like edema with 


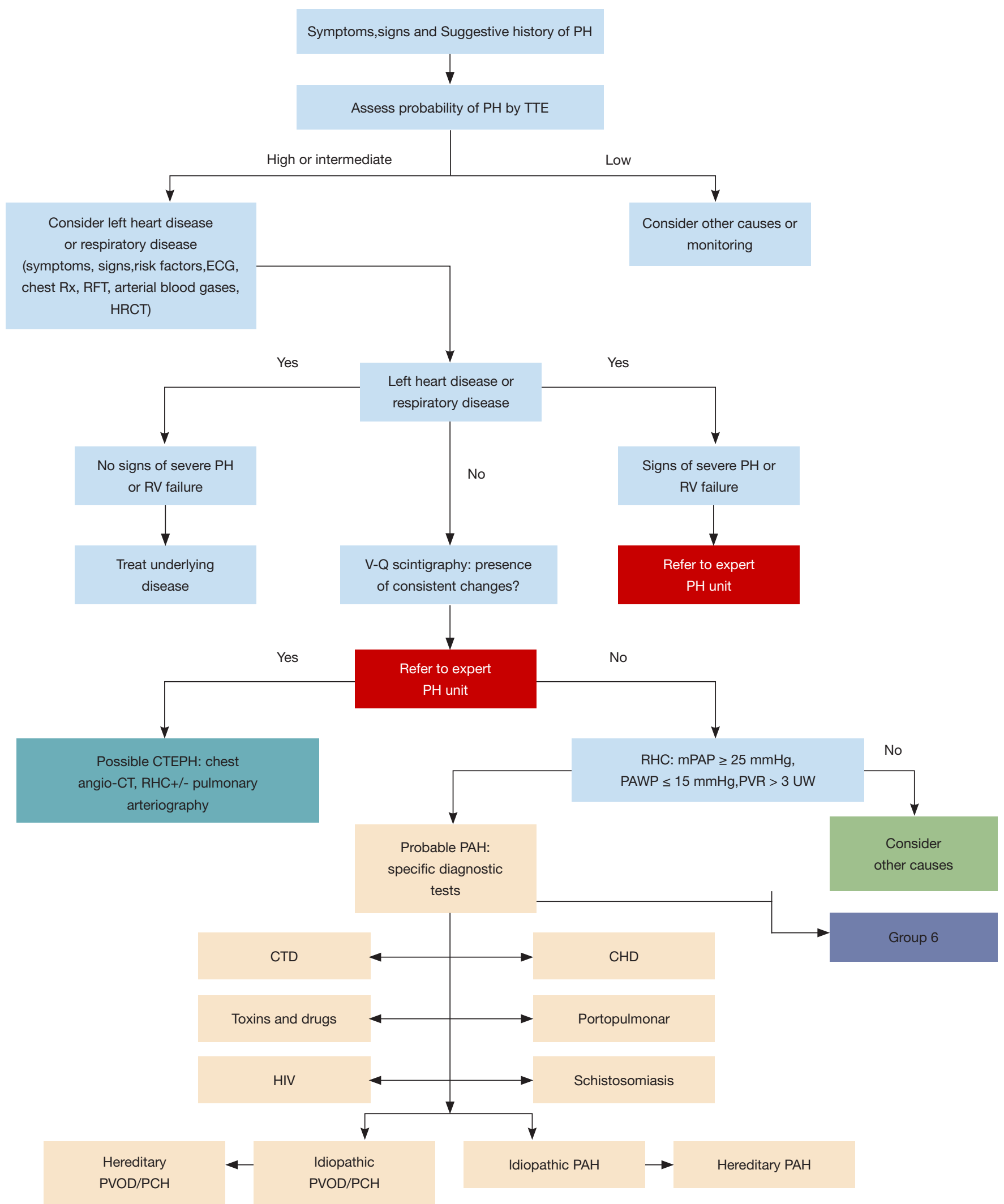

Figure 7 Adapted from 2015 ESC/ERS guidelines for the diagnosis and treatment of pulmonary hypertension algorithm guidelines. 
close monitoring of the patients' systemic blood pressure. Anticoagulants may also be initiated in patients with chronic thromboembolic pulmonary hypertension or patients with other comorbidities indicating anticoagulation e.g. atrial fibrillation, immobility, etc. $\mathrm{PH}$ patients are susceptible to pneumonia and should have once yearly influenza and pneumococcal vaccinations $(31,32,34-36)$.

Due to the pathophysiology behind $\mathrm{PH}$, involving increased pulmonary vascular resistance, vasodilator therapy through the use of calcium channel blockers (CCB) has proven an effective form of therapy for primary pulmonary hypertension and should only be initiated after a positive vasoreactivity study. Vasodilators not only dilate pulmonary vasculature they also dilate systemic vasculature and may end up worsening the patients' hemodynamic status due to systemic hypotension. Vigilant observation of patients' hemodynamic status is needed when initiating vasodilator therapy. Target-oriented therapies for $\mathrm{PH}$ also focus on the three main pathophysiological mechanisms that are responsible for the disease namely nitric oxide, endothelin, and prostacyclin. Prostacyclins such as epoprostenol activate the prostacyclin receptor which will ultimately lead to pulmonary vasodilation. They can be administered intravenously or inhaled. Inhaled prostacyclins are considered to be safer and well tolerated because they mainly act on pulmonary vascular pressure without affecting systemic vascular pressure. Endothelin-receptor antagonists such as bosentan, ambrisentan, and macitentan are a group of drugs that block endothelin receptors (ET1 and the ETA and ETB) that have been implicated in causing PAH by exerting pulmonary vasoconstriction and vascular remodeling. These can be given orally and are recommended for mild and moderate disease and for long time use. Phosphodiesterase 5 (PDE-5) inhibitors such as tadalafil, sildenafil, and vardenafil act on the nitric oxide pathway promoting vasodilatation and thereby decreasing the raised pulmonary arterial pressure. Prostacyclinreceptor agonists such as selexipag are potent vasodilators and inhibitors of platelet aggregation. These compounds bind to prostacyclin receptors on the surface of platelets and vascular smooth muscles which leads to the production of cyclic adenosine monophosphate, which induces relaxation of vascular smooth muscle (31,32,34-36).

\section{Sepsis and its cardiovascular effects}

Sepsis is a clinical syndrome invoked by the host's dysregulated immune response to a virulent organism leading to life-threatening organ dysfunction. In the past, sepsis was characterized by systemic immune response syndrome (SIRS), which involved two or more signs of SIRS (tachycardia, tachypnea, hypothermia, hyperthermia, or raised white blood cells) and a positive culture. This older method of diagnosis was revised in 2016 with the introduction of the sequential organ failure assessment score (SOFA score), which brought forth a new bedside scoring system known as the quick SOFA score (qSOFA). A positive diagnosis involves two of the following three using the qSOFA: respiratory rate $\geq 22 / \mathrm{min}$, change in the level of consciousness or altered mental status, and systolic blood pressure $<100 \mathrm{mmHg}(37,38)$. It should be noted that both the SOFA and qSOFA score is not a definitive diagnosis of sepsis and in situations where a patient fails to achieve a score of 2 or more should not completely rule out the possibility of sepsis or delay further investigation or needed medical intervention. In cases where there is a score of $<2$, further laboratory or imaging investigations should be carried out to either confirm or rule out any sepsisrelated organ dysfunction. In our case, after his physical examination, we noted that his qSOFA score was $<2$, with the only positive criteria being an increased respiratory rate; however, after further investigation, there was a definitive diagnosis of sepsis (39).

Patients with sepsis are considered high-risk patients with high morbidity and high mortality rates. The outcomes of sepsis are associated with an increase in organ dysfunction, the length of hospital stay, mortality, and finally an increase in chances of rehospitalization.

Sepsis affects the different organs of the body and has been associated with: (I) circulatory dysfunction through vascular dysfunction (due to vasodilation and increased vascular permeability) and cardiac dysfunction (sepsis has been characterized with myocardial depression leading to changes in both systolic and diastolic performances). (II) Pulmonary dysfunction due to injury of the pulmonary vasculature and increased microvascular permeability causing interstitial and alveolar edema which finally results in ARDS. (III) Renal dysfunction is a common cause of acute kidney injury or AKI. (IV) Sepsis-induced coagulopathy can cause upregulation of the coagulation cascade causing a state of hypercoagulability and an increase in thrombus formation or down-regulation causing disseminated intravascular coagulation (DIC). (V) Central nervous system dysfunction due to sepsis has been linked to sepsis-induced encephalopathy. Sepsis-induced coagulopathy also increases the chances of ischemic stroke 
$(38,40)$.

Despite the highlighted organ dysfunction, other factors affect patients' outcomes. Increased hospital length of stay not only depletes the patients' resources it also leaves the patients prone to acquiring other hospital-acquired infections $(41,42)$. Patients with sepsis have a higher average mortality rate; this has also been linked to continue even post-discharge. Mortality is further increased due to rehospitalization secondary to the complications that come about due to sepsis (43-45).

Sepsis-induced cardiac dysfunction (SICD) is a common effect of sepsis. Sepsis usually causes decreased vascular resistance, vasodilation, and increased permeability to the systemic vasculature system, which in turn causes an increase in the cardiac output and decrease in the left ventricular ejection fraction. SICD has been attributed to several mediators such as pro-inflammatory cytokines-(TNF- $\alpha$ )tumor necrosis factor, interleukin (IL-1 $\beta$ ), chemokines, increased production in nitric oxide, overstimulation of the sympathetic nervous system due to elevated endogenous catecholamines, mitochondria dysfunction leading to cytopathic hypoxia, changes in, ATP and glucose and fatty acid break down in the myocytes (46-50).

It is a common occurrence for septic patients to be hypotensive; however, that is not always the case. Our patient presented with systemic hypertension, with the pressure falling at around 190/110 $\mathrm{mmHg}$. After treatment, the high blood pressure resolved, and he has remained normotensive since then. Initially, we ruled out all the secondary causes of hypertension. The hypertension was due to the sepsis. Although cases of sepsis-induced hypertension have been reported and documented before. There has only been a little light shed on the specific pathophysiology and its relevant management (51).

\section{Conclusions}

We report an unusual case of a patient we were managing for severe pneumonia, sepsis, and $\mathrm{PH}$ with cardiac dysfunction. The cardiac dysfunction may be attributed to both the direct effect of sepsis on the cardiovascular system or by $\mathrm{PH}$.

\section{Acknowledgments}

Funding: This work was supported by 345 Talent Project of
Shenjing Hospital.

\section{Footnote}

Reporting Checklist: The authors have completed the CARE reporting checklist. Available at http://dx.doi.org/10.21037/ apm-20-1198

Conflicts of Interest: All authors have completed the ICMJE uniform disclosure form (available at http://dx.doi. org/10.21037/apm-20-1198). The authors have no conflicts of interest to declare.

Ethical Statement: The authors are accountable for all aspects of the work in ensuring that questions related to the accuracy or integrity of any part of the work are appropriately investigated and resolved. All procedures performed in studies involving human participants were in accordance with the ethical standards of the institutional and/or national research committee(s) and with the Helsinki Declaration (as revised in 2013). Written informed consent was obtained from the patient's father for publication of this case report and any accompanying images. A copy of the written consent is available for review by the Editor-inChief of the journal upon request.

Open Access Statement: This is an Open Access article distributed in accordance with the Creative Commons Attribution-NonCommercial-NoDerivs 4.0 International License (CC BY-NC-ND 4.0), which permits the noncommercial replication and distribution of the article with the strict proviso that no changes or edits are made and the original work is properly cited (including links to both the formal publication through the relevant DOI and the license). See: https://creativecommons.org/licenses/by-nc-nd/4.0/.

\section{References}

1. Arslan S, Ugurlu S, Bulut G, The association between plasma D-dimer levels and community-acquired pneumonia. Clinics (Sao Paulo) 2010;65:593-7.

2. Brown G. Coagulation cascade in sepsis: getting from bench to bedside? Crit Care 2003; 7:117-8.

3. Quinton, Lee J, Mizgerd JP. Dynamics of lung defense in pneumonia: resistance, resilience, and remodeling. Annu Rev Physiol 2015;77:407-30. 
4. Moldoveanu B, Otmishi P, Jani P, et al. Inflammatory mechanisms in the lung. J Inflamm Res 2009;2:1-11.

5. Dumas G, Lemiale V, Demoule A, et al. Improving survival in immunocompromised patients with hypoxemic acute respiratory failure. Ann Transl Med 2019;7:S293.

6. Mizgerd JP. Pathogenesis of severe pneumonia: advances and knowledge gaps. Curr Opin Pulm Med 2017;23:193-7.

7. Bajantri B, Venkatram S, Diaz-Fuentes G. Mycoplasma pneumoniae: A Potentially Severe Infection. J Clin Med Res 2018;10:535-44.

8. Aliberti S, Tobaldini E, Giuliani F, et al. Cardiovascular autonomic alterations in hospitalized patients with community-acquired pneumonia. Respir Res 2016;17:98.

9. Saraya T, Kurai D, Nakagaki K, et al. Novel aspects on the pathogenesis of Mycoplasma pneumoniae pneumonia and therapeutic implications. Front Microbiol 2014;5:410.

10. Corrales-Medina VF, Alvarez KN, Weissfeld LA, et al. Association between hospitalization for pneumonia and subsequent risk of cardiovascular disease. JAMA 2015;313:264-74.

11. Corrales-Medina VF, Musher Daniel M, Wells George A, et al. Cardiac Complications in Patients With CommunityAcquired Pneumonia. Circulation 2012;125:773-81.

12. Sakao S, Tatsumi K, Voelkel NF. Reversible or irreversible remodeling in pulmonary arterial hypertension. Am J Respir Cell Mol Biol 2010;43:629-34.

13. Pesto S, Begic Z, Prevljak S, et al. Pulmonary Hypertension - New Trends of Diagnostic and Therapy. Med Arch 2016;70:303-7.

14. Humbert M, Guignabert C, Bonnet S, et al. Pathology and pathobiology of pulmonary hypertension: state of the art and research perspectives. Eur Respir J 2019;53:1801887.

15. Kumar A, Neema PK. Severe pulmonary hypertension and right ventricular failure. Indian J Anaesth 2017;61:753-9.

16. Montani D, Günther S, Dorfmüller P, et al. Pulmonary arterial hypertension. Orphanet J Rare Dis 2013;8:97.

17. Ventetuolo CE, Klinger JR. Management of acute right ventricular failure in the intensive care unit. Ann Am Thorac Soc 2014;11:811-22.

18. Geovanini GR, Lorenzi-Filho G. Cardiac rhythm disorders in obstructive sleep apnea. J Thorac Dis 2018;10:S4221-30.

19. Singanayagam A, Singanayagam A, Elder DHJ, et al. Is community-acquired pneumonia an independent risk factor for cardiovascular disease? Eur Respir J 2012;39:187.

20. Rae N, Finch S, Chalmers JD. Cardiovascular disease as a complication of community-acquired pneumonia. Curr Opin Pulm Med 2016;22:212-8.
21. Eurich DT, Marrie TJ, Minhas-Sandhu JK, et al. Risk of heart failure after community acquired pneumonia: prospective controlled study with 10 years of follow-up. BMJ 2017;356:j413.

22. Sarkar M, Niranjan N, Banyal PK. Mechanisms of hypoxemia (published correction appears in Lung India 2017 Mar-Apr;34(2):220). Lung India 2017;34:47-60.

23. Lumb AB, Slinger P. Hypoxic Pulmonary Vasoconstriction: Physiology and Anesthetic Implications. Anesthesiology 2015;122:932-46.

24. Bhattacharya PT, Sharma S. Right Ventricular Hypertrophy. (Updated 2019 Apr 5). In: StatPearls (Internet). Treasure Island (FL): StatPearls Publishing; 2019 Jan-. Available online: https://www.ncbi.nlm.nih.gov/ books/NBK499876/

25. Bazan IS, Fares WH. Pulmonary hypertension: diagnostic and therapeutic challenges. Ther Clin Risk Manag 2015;11:1221-33.

26. Yealy DM, Huang DT, Delaney A, et al. Recognizing and managing sepsis: what needs to be done? BMC Med 2015;13:98.

27. Grignola JC, Domingo E. Acute Right Ventricular Dysfunction in Intensive Care Unit. BioMed Res Int 2017;2017:8217105.

28. Balegadde AV, Vijan V, Thachathodiyl R. A Case Series of Young Patients with Completely Reversed Severe Pulmonary Hypertension. J Clin Diagn Res 2017;11:OR04-5.

29. Rich JD, Rich S. Clinical diagnosis of pulmonary hypertension. Circulation 2014;130:1820-30.

30. Frost A, Badesch D, Gibbs JSR, et al. Diagnosis of pulmonary hypertension. Eur Respir J 2019;53:1801904.

31. van Wolferen SA, Grünberg K, Vonk Noordegraaf A. Diagnosis and management of pulmonary hypertension over the past 100 years. Respir Med 2007;101:389-98.

32. Haeck MLA, Vliegen HW. Diagnosis and treatment of pulmonary hypertension. Heart 2015;101:311.

33. Galiè N, Humbert M, Vachiery JL, et al. 2015 ESC/ERS Guidelines for the diagnosis and treatment of pulmonary hypertension: The Joint Task Force for the Diagnosis and Treatment of Pulmonary Hypertension of the European Society of Cardiology (ESC) and the European Respiratory Society (ERS): Endorsed by: Association for European Paediatric and Congenital Cardiology (AEPC), International Society for Heart and Lung Transplantation (ISHLT). Eur Respir J 2015;46:903-75.

34. Corris P, Degano B. Severe pulmonary arterial hypertension: treatment options and the bridge to 
transplantation. Eur Respir Rev 2014;23:488.

35. Raja SG, Raja SM. Treating pulmonary arterial hypertension: current treatments and future prospects. Ther Adv Chronic Dis 2011;2:359-70.

36. Huang HB, Xu B, Liu GY, et al. N-terminal pro-Btype natriuretic peptide for predicting fluid challenge in patients with septic shock. Ann Transl Med 2019;7:264.

37. Hotchkiss RS, Moldawer LL, Opal SM, et al. Sepsis and septic shock. Nat Rev Dis Primers 2016;2:16045.

38. Fujishima, S. Organ dysfunction as a new standard for defining sepsis. Inflamm Regen 2016;36:24.

39. Singer M, Deutschman CS, Seymour CW, et al. The Third International Consensus Definitions for Sepsis and Septic Shock (Sepsis-3). JAMA 2016;315:801-10.

40. Caraballo C, Jaimes F. Organ Dysfunction in Sepsis: An Ominous Trajectory From Infection To Death. The Yale Journal of Biology and Medicine 2019;92:629-40.

41. Glied S, Cohen B, Liu J, et al. Trends in mortality, length of stay, and hospital charges associated with health careassociated infections, 2006-2012. Am J Infect Control 2016;44:983-9.

42. Barnett AG, Page K, Campbell M, et al. The increased risks of death and extra lengths of hospital and ICU stay from hospital-acquired bloodstream infections: a casecontrol study. BMJ Open 2013;3:e003587.

43. Martin GS. Sepsis, severe sepsis and septic shock: changes in incidence, pathogens and outcomes. Expert Rev Anti Infect Ther 2012;10:701-6.

Cite this article as: Gitonga EN, Wang J, Yu S, Wu N, Shen H. Left ventricular dysfunction and reversible pulmonary hypertension secondary to severe pneumonia in a background of sepsis: a case report and review of the literature. Ann Palliat Med 2020;9(5):3629-3642. doi: 10.21037/apm-20-1198
44. Rahmel T, Schmitz S, Nowak H, et al. Long-term mortality and outcome in hospital survivors of septic shock, sepsis, and severe infections: The importance of aftercare. PLoS One 2020;15:e0228952.

45. Shankar-Hari M, Rubenfeld GD. Understanding LongTerm Outcomes Following Sepsis: Implications and Challenges. Curr Infect Dis Rep 2016;18:37.

46. Romero-Bermejo FJ, Ruiz-Bailen M, Gil-Cebrian J, et al. Sepsis-induced cardiomyopathy. Curr Cardiol Rev 2011;7:163-83.

47. Liu YC, Yu MM, Shou ST, et al. Sepsis-Induced Cardiomyopathy: Mechanisms and Treatments. Front Immunol 2017;8:1021.

48. Drosatos K, Lymperopoulos A, Kennel PJ, et al. Pathophysiology of sepsis-related cardiac dysfunction: driven by inflammation, energy mismanagement, or both? Curr Heart Fail Rep 2015;12:130-40.

49. Kakihana Y, Ito T, Nakahara M, et al. Sepsis-induced myocardial dysfunction: pathophysiology and management. J Intensive Care 2016;4:22.

50. Rosenkranz S, Gibbs JS, Wachter R, et al. Left ventricular heart failure and pulmonary hypertension. Eur Heart J 2016;37:942-54.

51. Saleh M. Sepsis-related hypertensive response: friend or foe? BMJ Case Rep 2014;2014:bcr2014204610.

(English Language Editor: J. Chapnick) 